\title{
A Terapia Cognitivo-Comportamental Aplicada ao CAPS ad: Uma Revisão
}

\author{
de Escopo \\ Érica Aparecida Schefer de Almeida* \\ Universidade Federal de Juiz de Fora - UFJF, Juiz de Fora, MG, Brasil \\ ORCID: https://orcid.org/0000-0003-0155-2897 \\ Laisa Marcorela Andreoli Sartes** \\ Universidade Federal de Juiz de Fora - UFJF, Juiz de Fora, MG, Brasil \\ ORCID: https://orcid.org/0000-0002-1335-4305
}

\section{RESUMO}

Este estudo tem como objetivo realizar uma revisão de escopo sobre estratégias cognitivas e/ou comportamentais empregadas no tratamento de dependência de álcool e outras drogas no mundo, assim como levantar estudos sobre o uso destas abordagens em CAPS ad no Brasil. Foram levantados artigos de revisão sobre "estratégias cognitivas ou comportamentais para tratamento de dependência de álcool e outras drogas" e publicações com o tema "uso de terapias cognitivas e/ou comportamentais em CAPS ad". Foram encontradas apenas cinco publicações relatando emprego de terapias cognitivas e comportamentais em CAPS ad. Estes estudos descreveram intervenções em grupos coordenados por psicólogos, enfermeiros ou outros profissionais de nível superior. Foram incluídos ainda 23 artigos de revisão sobre resultados de tratamentos de base cognitivo-comportamental para tratamento de dependência de álcool e outras drogas. Conclui-se que as estratégias cognitivo-comportamentais apresentam várias características vantajosas à saúde pública, como a possibilidade de sistematização em protocolos, o que facilita sua aplicação e avaliação de resultados terapêuticos. No entanto, persistem obstáculos para adoção desta abordagem nos serviços de saúde mental no Brasil, sendo então necessário avançar no desenvolvimento de instrumentos de políticas públicas que permitam a difusão de boas práticas de intervenções comprovadamente custo-efetivas e acessíveis à população.

Palavras-chave: centro de atenção psicossocial, serviços de saúde mental, transtornos relacionados ao uso de substâncias, terapia cognitiva, terapia comportamental. 


\title{
The Cognitive-Behavioral Therapy Applied to CAPS ad: A Scope Review
}

\begin{abstract}
This study aims to conduct an escope review on the use of cognitive-behavioral approaches to treatment of alcohol and other drug addiction in CAPS ad, as well as find studies on the use of this approach in CAPS ad in Brazil. We conducted searches with the theme "use of cognitive and / or behavioral therapies in CAPS ad" and review articles on "cognitive or behavioral strategies for treatment of alcohol and other drug dependence". Only five publications were found reporting the use of cognitive and behavioral therapies in CAPS ad. These studies described interventions in groups coordinated by psychologists, nurses or other graduated professionals. Twenty-three review articles on outcomes of cognitive-behavioral treatments for addiction treatment of alcohol and other drugs were also included. We concluded that cognitive-behavioral strategies showcase several advantageous characteristics to public health, such as the possibility of systematization in protocols, which facilitates their application and evaluation of therapeutic results. However, obstacles to the adoption of this approach in mental health services in Brazil persist, so it is necessary to advance in developing public policy instruments that allow the dissemination of good practices in interventions that are proven to be cost-effective and accessible to the population.
\end{abstract}

Keywords: psychosocial care center, mental health services, substance use disorders, cognitive therapy, behavioral therapy.

\section{La Terapia Cognitivo-Conductual Aplicada al CAPS ad: Una Revisión del}

\begin{abstract}
Alcance
RESUMEN

Este estudio tiene como objetivo realizar una revisión del alcance sobre el empleo de abordajes cognitivo-conductuales para tratamiento de dependencia de alcohol y otras drogas en los CAPS ad en Brasil. Se levantaron publicaciones con el tema "uso de terapias cognitivas y/o conductuales en CAPS ad" y artículos de revisión sobre "estrategias cognitivas o conductuales para el tratamiento de la dependencia del alcohol y otras drogas". Se encontraron sólo cinco publicaciones que relatan empleo de terapias cognitivas y conductuales en CAPS ad. Estos estudios describieron intervenciones en grupos coordinados por psicólogos, enfermeros u otros profesionales de nivel superior. Se incluyeron 23 artículos de revisión sobre resultados de tratamientos de base cognitivo-conductual para tratamiento de dependencia de alcohol y otras drogas. Se concluye que las estrategias cognitivo-conductuales presentan varias características ventajosas para la salud pública, como la posibilidad de sistematización en protocolos, lo que facilita su aplicación y evaluación de resultados terapéuticos. Sin embargo, persisten obstáculos para la adopción de este enfoque en los servicios de salud mental en Brasil, por lo que es necesario avanzar en el desarrollo de instrumentos de política pública que permitan la difusión de buenas prácticas de intervenciones que demuestren ser costo-efectivas y accesibles a la población.
\end{abstract}

Palabras clave: centro de atención psicosocial, servicios de salud mental, trastornos relacionados con el uso de sustancias, terapia cognitiva, terapia conductual. 
A política do Ministério da Saúde (2004) para a Atenção Integral a Usuários de álcool e outras drogas contempla que a assistência a pessoas com problemas relacionados ao uso de substâncias deve ser oferecida em todos os níveis de atenção, privilegiando os cuidados em dispositivos extra-hospitalares, como o Centro de Atenção Psicossocial para álcool e drogas (CAPS ad). O CAPS ad é um serviço da Rede de Atenção Psicossocial (RAPS) específico para o cuidado, atenção integral e continuada às pessoas com necessidades em decorrência do uso dessas substâncias (Portaria n. 3.088, 2011).

No campo do tratamento da dependência de substâncias psicoativas, as abordagens cognitivo-comportamentais apresentam grande número de resultados de pesquisas demonstrando sua eficácia e estão entre as intervenções mais indicadas para tratamento destes problemas (McGovern \& Carroll, 2003).

A formação dos profissionais que participaram dos primeiros serviços propostos pela Reforma Psiquiátrica (RP), entre eles os CAPS, teve grande contribuição da Psicanálise, o que justifica a predominância deste referencial nestes dispositivos e a extensa produção teórica na área da Saúde Mental sobre esse enfoque (Ferreira Neto, 2008). A inserção das Terapias Cognitivas (TC) nos currículos das faculdades de Psicologia e na Saúde Pública no Brasil é recente, sobretudo nas cidades de interior (Rangé, Falcone, \& Sardinha, 2007).

As abordagens cognitivo-comportamentais podem ser definidas pelos termos "Terapia cognitivo-comportalmental (TCC)", "Terapia Comportamental" e "Terapias Cognitivas", variando conforme autores e momento histórico (geração de terapias cognitivas). Alguns autores como Knapp (2004), empregam o termo genérico TCC para se referir a todo movimento de terapias cognitivo-comportamentais, que abrange mais de 20 abordagens (incluindo o movimento de Terceira Onda). Outros autores, como os terapeutas de Terceira Geração, julgam mais apropriado o termo Terapia Comportamental para incluir as chamadas "três gerações", uma vez que o termo TCC é frequentemente utilizado para se referir especificamente a tratamentos que incluem estratégias de reestruturação cognitiva (LucenaSantos, Pinto-Gouveia, \& Oliveira, 2015).

Apesar de não existir uma definição geral que possa descrever a abordagem de forma adequada e concisa, existem características centrais, sendo considerada científica, ativa, focada no presente, focada na aprendizagem, individualizada, progressiva e breve (LucenaSantos et al., 2015). Dobson e Scherrer (2011) destacam três proposições fundamentais no cerne das terapias cognitivo-comportamentais: a atividade cognitiva influencia o comportamento; a atividade cognitiva pode ser monitorada e alterada; e o comportamento desejado pode ser influenciado mediante mudança cognitiva. 
Os sistemas de saúde em todo mundo têm se esforçado para conter custos e melhorar a relação custo-benefício dos tratamentos de saúde mental, procurando identificar e implementar tratamentos eficazes e limitados pelo tempo (Dobson \& Scherrer, 2011). O conceito de "Práticas Baseadas em Evidências" (PBE) é uma tendência que tem ganhado adeptos em diversas áreas da saúde, impactando o planejamento e implementação de políticas e práticas para a abordagem de substâncias psicoativas (Bello, 2015).

A TCC foi avaliada nos principais projetos de experimentos controlados e randomizados para estudos de tratamento de dependência de drogas, como o Projeto MATCH (Matching Alcoholism Treatment to Client Heterogeneity) realizado pelo National Institute on Alcohol Abuse and Alcoholism (NIAAA) dos Estados Unidos para pacientes dependentes de álcool e o "Collaborative Cocaine Treatment Study" realizado pelo National Institute on Drug Abuse (NIDA). Em ambos projetos foi efetiva na redução do consumo de álcool e outras drogas e no apoio à melhoria dos demais aspectos da vida do usuário (Project MATCH Research Group, 1997; Siqueland \& Crits-Christoph, 1999).

Silva, Pereira e Aquino (2011) consideram que as abordagens cognitivas e comportamentais têm se mostrado como uma ferramenta valiosa na saúde pública, por respaldar uma série de programas e intervenções que podem ser eficazes e adequadas à realidade, com todas as suas particularidades, como tempo reduzido e elevada demanda.

Considerando a necessidade de práticas baseadas em evidências no tratamento de substâncias psicoativas, bem como os resultados da eficiência e eficácia dos tratamentos de base cognitiva e/ou comportamental e seu crescimento nos serviços públicos, interroga-se sobre o emprego destas abordagens para tratamento de dependência de álcool e outras drogas em CAPS ad no Brasil. Este estudo tem como objetivo realizar uma revisão de escopo sobre abordagens cognitivo-comportamentais empregadas no tratamento de dependência de álcool e outras drogas e levantar estudos sobre o uso destas estratégias nos CAPS ad.

\section{Método}

Trata- se de uma revisão de escopo com os seguintes objetivos: 1) levantar estudos sobre resultados do uso de abordagens cognitivo e/ou comportamentais para tratamento de dependência de álcool e outras drogas no mundo; 2) levantar publicações que relatem uso de terapias cognitivas e/ou comportamentais no tratamento de dependentes de álcool e outras drogas em CAPS ad no Brasil. 
Inicialmente foram realizadas buscas referentes ao primeiro objetivo utilizando os descritores Mesh "Mental Health Services" AND "Cognitive Therapy" AND "Behavior Therapy" AND "Substance-Related Disorders", nas bases de dados Pubmed, Scopus e Psycinfo, sendo encontrados 224 resultados no total. Também foi realizada uma pesquisa na base Pubmed com os descritores "cognitive therapy" OR "behavior therapy" AND "substance-related disorders" AND "treatment outcome" totalizando 263 artigos.

Em seguida, de forma a atender o segundo objetivo foram definidos os seguintes descritores chave, por meio da base de dados Bireme (BVS): "Centros de atenção psicossocial", "serviços de saúde mental", "terapia cognitiva" e "terapia comportamental", cujas combinações resultaram em 832 documentos. Também foram realizadas pesquisas nas bases "Periódicos CAPES" e "Google acadêmico", com as combinações "centro de atenção psicossocial" AND "terapia cognitivo-comportamental" AND "dependência de drogas", sendo encontrados 1.407 artigos.

Todas as publicações encontradas nas diferentes bases de dados foram exportadas para o Endnote, totalizando 2726 estudos. Após a exclusão de duplicatas, restaram 1.990 periódicos, que foram submetidos aos seguintes critérios de inclusão: artigos nos idiomas português, inglês e espanhol, que abordassem como tema "uso de estratégias cognitivas ou comportamentais para tratamento de dependência de álcool e outras drogas" ou "uso de terapias cognitivas ou comportamentais em Centros de Atenção Psicossocial”. Os artigos foram analisados por meio de leitura de títulos e resumos.

Após os artigos serem avaliados por estes critérios, foram reduzidos para 97 estudos que foram então analisados por meio de leitura de resumo ou artigo na íntegra, quando necessário. Foram mantidos somente artigos de revisão sobre resultados de intervenções cognitivas ou comportamentais para tratamento de dependência de álcool e outras drogas e artigos empíricos e dissertações, que tivessem como tema o uso de terapias cognitivas e comportamentais em CAPS ad, resultando 28 artigos.

\section{Resultados}

Quanto ao primeiro objetivo, os artigos que se tratavam de revisões de literatura sobre tratamentos cognitivos ou comportamentais de dependência de álcool e outras drogas (23 artigos) apresentaram os seguintes eixos temáticos: Tratamentos Baseados em Evidências; Entrevista Motivacional/Terapia de Aprimoramento Motivacional; Manejo de Contingências; Prevenção de Recaídas; Treino de Habilidades Sociais (THS); Terapia de Exposição; 
Tratamento por Reforço Comunitário; Terapia Comportamental de Casal; Terapias Baseadas em Mindfulness e Terapia Cognitivo-Comportamental pela Internet.

Por meio de análise temática, os resultados encontrados nestes artigos foram agrupados, gerando as seguintes categorias: 1) Técnicas comportamentais aplicadas à dependência de álcool e outras drogas; 2) A Terapia cognitivo-comportamental aplicada à dependência de álcool e outras drogas; 3) Modelo de Prevenção de Recaídas e Prevenção de Recaída Baseada em Mindfulness; 4) Entrevista Motivacional.

Em relação ao segundo objetivo, os artigos sobre tratamento com terapias cognitivas e comportamentais em CAPS ad, foram encontradas cinco publicações. Destes estudos, dois estavam no formato de dissertação e os outros três no formato de artigo. Dois estudos apresentaram pesquisas qualitativas descritivas exploratórias, dois artigos descreveram relatos de intervenção e um artigo se tratou de um projeto de intervenção, sem apresentação de resultados. Duas publicações foram desenvolvidas por profissionais de enfermagem, duas por psicólogos e uma dissertação foi elaborada por um terapeuta ocupacional com especialização em Saúde Pública.

\section{Teorias e Técnicas Comportamentais Aplicadas à Dependência de Álcool e Outras Drogas}

A teoria comportamental se concentra em estratégias para modificação e melhora do estado motivacional, explorando comportamentos associados ao uso de drogas, com objetivo de mudar o estilo de vida e os comportamentos de risco para o uso (Silva \& Serra, 2004). Conforme Miguel e Gaya (2013), a perspectiva comportamental para a dependência de substâncias psicoativas pode ser dividida em duas correntes teóricas: 1) focadas no condicionamento clássico ou respondente; 2) focadas no condicionamento operante.

O condicionamento clássico foi identificado por Pavlov e demonstra a relação entre a exposição de pacientes a determinadas situações, lugares, objetos e pessoas (estímulos) e a reação deles a estes sinais (resposta condicionada). Este processo está presente no desenvolvimento e manutenção da dependência de substâncias psicoativas e os tratamentos baseados nesta forma de condicionamento são focados na extinção das respostas condicionadas (Silva \& Serra, 2004).

A técnica desenvolvida para promover tais procedimentos pode ser traduzida para o português como "tratamento por exposição a estímulos" (CET - cue exposure treatment). A CET envolve exposição controlada e repetida a estímulos condicionados, sem o uso da 
substância, com o objetivo de reduzir as respostas condicionadas da fissura pela extinção e habituação. Os indivíduos dependentes de álcool e outras drogas são expostos a gatilhos, como a visão e o cheiro de uma bebida favorita, sem consumir álcool (Roggi, Gama, Neves, \& Garcia, 2015).

Os estudos de Jhanjee (2014) e Roggi et al. (2015) apontaram o CET como uma abordagem eficaz na redução da fissura. No entanto, foram verificadas limitações na sua aplicação, uma vez que os resultados foram limitados na promoção da abstinência e não se mantiveram ao longo do tempo.

A teoria do Condicionamento Operante foi desenvolvida por Skinner, quem demonstrou que a manutenção de um comportamento se dá pela presença de um reforço. $\mathrm{O}$ reforço consiste em qualquer estímulo ou evento que aumente a probabilidade de ocorrência de um comportamento. O usuário de substâncias psicoativas tem o aumento de seu uso devido às propriedades reforçadoras das substâncias psicoativas que provocam uma retroalimentação do consumo. Exemplos de tratamentos que envolvem condicionamento operante são a Terapia Comportamental de Casal, o Tratamento por Reforço Comunitário e o Manejo de Contingências (Miguel \& Gaya, 2013).

O Manejo de Contingências (MC) ou terapia baseada em vouchers aplica os princípios do reforço positivo para o desempenho dos comportamentos consistentes com uma redução ou cessação do uso de drogas (Rawson \& Clark, 2017). Vários estudos (McGovern \& Carroll, 2003; Jhanjee, 2014; Rawson \& Clark, 2017) relataram que o emprego de MC no tratamento de transtornos de uso de substâncias promove aumento do período de abstinência com manutençaõ de resultados a longo prazo, em uma ampla gama de substâncias. No entanto, a intervenção não tem sido amplamente utilizada na prática clínica, devido aos altos custos percebidos de provisão de tais intervenções (McGovern \& Carroll, 2003; Jhanjee, 2014; Rawson \& Clark, 2017).

O Tratamento por Reforço Comunitário (CRA - "Community Reinforcement Approach") é uma abordagem biopsicossocial multifacetada, que tem como objetivo fazer mudanças no estilo de vida de abuso de substâncias. Ela reconhece o papel do ambiente no abuso habitual, e concentra-se em desenvolver recursos positivos alternativos no ambiente social do usuário. Roozen et al. (2004) realizaram uma revisão sistemática com o objetivo de avaliar a eficácia do CRA no tratamento de álcool, cocaína e dependência de opiáceos e verificaram apenas evidências moderadas em relação ao impacto de tratamento com CRA sem outras abordagens adicionais. 
Baseada nos princípios comportamentais, a Terapia Comportamental de Casal (BCT Behavior Couples Therapy) sustenta que interações familiares disfuncionais produzem contingências que reforçam o consumo de álcool e outras substâncias. O'Farrell, Fals-Stewart e colegas desenvolveram a BCT a partir do reconhecimento de que o alcoolismo é um problema sistêmico, afetando o casal, relacionamentos, filhos, família, amigos e sociedade (O'Farrell \& Fals-Stewart, 2006).

Estudos de McGovern e Carroll (2003) e Rawson \& Clark (2017) constatam que a BCT para o tratamento de dependência de álcool e outras drogas apresenta vários resultados positivos, como aumento do período de abstinência, melhora do relacionamento do casal e redução da violência doméstica. Foram verificadas limitações, como falta de avaliação empírica, considerando a influência de fatores externos e de condução destes em outros ambientes além de clínicas comunitárias.

\section{A Terapia Cognitivo-Comportamental Aplicada à Dependência De Álcool e Outras Drogas}

Conforme o Modelo Cognitivo de Abuso de Substâncias de Beck, certos indivíduos desenvolveram uma vulnerabilidade cognitiva ao abuso de drogas. Crenças e desejos relativos ao uso de drogas são normalmente ativados em circustâncias de alto risco particulares e previsíveis, que podem ser internas ou externas. As circunstâncias internas incluem estado emocional desconfortável, como depressão, ansiedade e tédio. Exemplos de circunstâncias externas podem ser uma festa onde drogas estão sendo usadas (Beck, Wright, Newman, \& Liese, 1993).

Conforme Freire (2011), o tratamento da dependência de drogas com base cognitivocomportamental compreende três fases. $\mathrm{Na}$ fase inicial são aplicadas intervenções comportamentais e motivacionais. A fase intermediária compreende psicoeducação quanto ao modelo cognitivo e quanto aos transtornos por uso de substâncias. Também incluem técnicas específicas de reestruturação cognitiva e mudança de hábitos. A consolidação deste aprendizado pode ser considerada a fase final do tratamento, com intervenções especificamente estruturadas para que o paciente desenvolva autonomia e a manutenção da abstinência deve ser monitorada.

Múltiplas meta-análises e revisões sistemáticas verificaram que a TCC é eficaz para o tratamento da dependência de vários tipos de substâncias. Também foi constatado que tratamentos que combinam TCC com outra abordagem comportamental obtiveram tamanho 
de efeito maior do que tratamento com TCC associado a tratamento farmacológico ou TCC isoladamente (Jhanjee, 2014; McGovern \& Carroll, 2003; Rawson \& Clark, 2017; Roggi et al., 2015).

\section{Modelo de Prevenção de Recaídas e Prevenção de Recaída Baseada em Mindfulness}

O modelo de Prevenção de Recaídas (PR), desenvolvido por Marlatt e Gordon, tem como base a psicologia social cognitiva, associada a um modelo conceitual dos episódios de recaídas e um conjunto de estratégias cognitivo-comportamentais para preveni-los. Um aspecto central do modelo é a classificação detalhada de fatores ou situações que podem contribuir para episódios de recaída (Larimer, Palmer, \& Marlatt, 1999).

Recentemente, Alan Marlatt desenvolveu, juntamente com sua equipe, o programa Prevenção de Recaída Baseada em Mindfulness (MBRP - Mindfulness Based Relapse Prevention) que integra habilidades do modelo Prevenção de Recaída (RP) e treinamento em práticas de meditação Mindfulness (Bowen et al., 2014). O MBRP é um programa ambulatorial adjunto ao tratamento de dependência química que foi projetado para ajudar os clientes a aumentarem a consciência sobre os gatilhos, experiências físicas, emocionais e cognitivas, aprendendo a responder habilmente, ao invés de reagir automaticamente (Boewn et al., 2014).

Os estudos de Bowen et al. (2014) e os estudos de Jhanjee (2014) apontam que o modelo de Prevenção de Recaídas e a Prevenção de Recaídas Baseada em Mindfulness têm uma base de evidência considerável no tratamento de distúrbios de uso de substâncias, possibilitando melhora das habilidades necessárias para lidar com as situações de alto risco e diminuição dos episódios de recaída. Além disso, a associação com Mindfulness fortalece a capacidade de monitorar e lidar habilmente com os desconfortos associados à fissura ou afetos negativos, possibilitando manutenção de resultados a longo prazo (Bowen et al., 2014; Chiesa \& Serretti, 2014).

\section{Entrevista Motivacional}

A Entrevista Motivacional (EM), também conhecida como Terapia de Aprimoramento Motivacional (MET - "Motivational enhancement therapy"), foi desenvolvida por Miller e Rollnick, inicialmente em 1983, como uma intervenção breve para o alcoolismo, na qual a motivação dos pacientes costuma ser um obstáculo para a mudança. A EM atua na própria 
motivação dos pacientes para a mudança e adesão ao tratamento. É um estilo clínico habilidoso para evocar dos pacientes as suas boas motivações para fazer mudanças comportamentais no interesse de sua própria saúde (Rollnick, Miller, \& Butler, 2009).

A eficácia da EM foi avaliada em meta-análises e estudos de revisão verificando melhora na aderência ao tratamento e na redução do consumo. Além disso, vários estudos investigaram a associação de intervenção breve de TCC com EM ou MET para tratamento de várias drogas, incluindo anfetaminas, cocaína e maconha, tendo como resultado a redução no consumo de drogas, bem como aumento do período de abstinência e de melhorias significativas nos estágios de mudança (McGovern \& Carroll, 2003; Rawson \& Clark, 2017).

\section{Aplicação de Abordagens Cognitivo-Comportamentais no CAPS Ad}

Santos (2010) abordou a temática do grupo como intervenção terapêutica no CAPS ad, realizando uma pesquisa qualitativa exploratório-descritiva, por meio de entrevistas semiestruturadas com 17 profissionais de nível superior, de três CAPS ad do Espírito Santo. O objetivo geral do estudo foi descrever as diversas atividades grupais desenvolvidas pelos profissionais e um dos objetivos específicos foi identificar a abordagem teórico-metodológica adotada nos grupos.

As atividades grupais relatadas no estudo consistem em grupos de acolhimento, acompanhamento, oficinas terapêuticas e assembleias. Em relação ao referencial teórico empregado nos grupos, foi observado que os profissionais não seguem uma única abordagem, mas diferentes metodologias como estratégias comportamentais, intervenção breve, prevenção de recaídas, arteterapia, terapia sistêmica, psicanálise, entre outras. Em relação à TCC, os profissionais que relataram empregá-la, a consideram "uma abordagem de fácil aplicação, diretiva e focalizada”, portanto, adequada a usuários de álcool e outras drogas (Santos, 2010).

Conforme o estudo, profissionais que atuam com grupos nos CAPS ad se utilizam de recursos e abordagens que, na maioria das vezes, são aprendidos na prática ou com base em sua formação acadêmica. A principal dificuldade indicada pelos participantes para o desenvolvimento de grupos foi a falta de uma fundamentação mínima sobre essa temática em sua graduação. Segundo a autora, esta lacuna presente na formação se deve a uma lógica de atenção reduzida ao espaço clínico do consultório, altamente prescritiva e centrada na doença, que não dá bases suficientes para o trabalho coletivo.

Foi destacada a importância da ampliação do conhecimento sobre o processo grupal, a fim de melhor preparar os profissionais para seu desenvolvimento nos serviços de saúde, visto 
que a estratégia grupal se coloca em lugar de destaque no "novo paradigma de saúde". Neste modelo de atenção, os grupos devem funcionar como espaços de reflexão, de interação, sendo facilitadores da mudança de estilos de vida, e não meramente espaços informativos, de acompanhamento e prescrição de informações (Santos, 2010).

Além da necessidade de capacitação dos profissionais para gerenciamento de grupos, foi apontada a urgência de sistematização dessas atividades. A falta de uma metodologia estruturada para desenvolvimento de trabalhos em grupo gera dificuldades de lidar com questões que emergem durante este processo, rotatividade dos participantes e a consequente desmotivação do profissional (Santos, 2010).

A temática das intervenções grupais também esteve presente em um estudo descritivoexploratório, desenvolvido por Bourguignon, Guimarães e Siqueira (2010), que teve como objetivo descrever a assistência prestada por três enfermeiros que atuam em grupos terapêuticos em três CAPS ad do Espírito Santo. Os grupos desenvolvidos por estes profissionais são semelhantes aos do estudo anterior (grupos de acolhimento, acompanhamento, oficinas e assembleias).

Quanto à abordagem teórico-metodológica adotada, observou-se a utilização do referencial cognitivo-comportamental e protocolos do Ministério da Saúde. Um dos entrevistados justificou a preferência por essa abordagem pela experiência anterior com TCC em grupos de tabagismo e por ser "uma abordagem diretiva e de fácil aplicação".

Os autores consideram que é necessário que o enfermeiro se aproprie da ferramenta grupal, uma vez que ela é a principal estratégia terapêutica empregada nos CAPS ad. No entanto, ainda é incipiente o tratamento desse conteúdo em sua formação, que embora preconize o cuidado do indivíduo em uma visão holística, ainda se constitui em um modelo centrado no doente e na doença (Bourguignon et al., 2010).

Kantorski, Lisboa e Souza (2005) relataram a experiência de um grupo chamado de "prevenção de recaídas", conduzido por dois enfermeiros e um psiquiatra, em um CAPS ad II, no período de dois anos. O grupo acontecia toda semana, com duração média de uma hora e meia. Os coordenadores do grupo utilizaram o protocolo de Prevenção de Recaídas proposto por Knapp e Bertolote (1994), em seu livro "Prevenção de Recaídas: um manual para pessoas com problemas pelo uso de álcool e de drogas". Durante os encontros realizados foram aplicados princípios da EM, PR e TCC.

Os autores consideram que a utilização da TCC associada a princípios motivacionais possibilitou o acolhimento das recaídas, bem como a ênfase na motivação para o tratamento. Foram estabelecidas metas de tempo de abstinência e foram feitas modificações para modos 
de vida mais realistas, que reforçam a autoeficácia e autoestima do paciente e contribuem na aderência ao tratamento (Kantorski et al., 2005).

No estudo de Romanini, Pereira e Dias (2016), foi apresentada uma breve revisão de literatura sobre o uso de substâncias, TCC e PR, e descrita uma proposta de grupo de prevenção de recaídas em um CAPS ad do Rio Grande do Sul. Foi apresentado apenas o programa a ser implementado, sem resultados. Tratava-se de um grupo fechado, estruturado, com dezesseis encontros, que aconteceriam duas vezes por semana e seria conduzido por psicólogos. Seriam empregadas estratégias como EM, PR, TCC e THS.

O estudo de Martins (2011) consiste em um relato de intervenção realizado em um grupo conduzido pela autora, no período de um ano, com pacientes dependentes de benzodiazepínicos em tratamento em um CAPS ad em Porto Alegre. A psicóloga coordenadora do grupo desenvolvia a atividade uma vez por semana, com duração de uma hora, empregando um protocolo de tratamento de base cognitivo-comportamental. O grupo inicialmente foi composto por 17 participantes, mas era aberto à entrada de novos integrantes durante o curso do tratamento. Algumas estratégias utilizadas no grupo foram: THS, PR, reestruturação cognitiva, treino de assertividade e relaxamento.

Foi verificado que seis dos dezessete participantes iniciais do grupo cessaram o uso de benzodiazepínicos. Além disso, a autora observou que a maioria dos pacientes alcançaram maior qualidade de vida e perceberam o grupo como um espaço para aliviar tensões e angústias. Foi destacado que pesquisas empíricas são necessárias para validar a abordagem cognitivo-comportamental em grupo para os problemas de abuso e dependência de benzodiazepínicos (Martins, 2011).

\section{Discussão}

As estratégias de base cognitivo-comportamental, sobretudo a TCC, PR e EM, são estruturadas, diretivas e focais. Em contraste com as abordagens psicodinâmicas que não apresentam evidências disponíveis, a TCC apresenta protocolos, o que facilita sua aplicação e avaliação de resultados terapêuticos. Além disso, são adequadas à saúde pública e podem ser incorporadas por qualquer profissional de saúde (Souza, 2010).

Apesar destas vantagens e dos inúmeros estudos relatando evidências da efetividade destas abordagens no tratamento de substâncias psicoativas, foram verificados apenas cinco estudos relatando aplicação de estratégias cognitivo-comportamentais em CAPS ad no Brasil, sendo que um deles se tratava de um projeto de intervenção sem apresentação de resultados. 
Dois estudos abordaram as estratégias cognitivo-comportamentais, sendo aplicadas por profissionais de nível superior nas atividades coletivas presentes nos CAPS. Os outros dois estudos também abordaram intervenções em grupo, porém, em um formato sistematizado, baseado em protocolos da abordagem Prevenção de Recaídas associado a técnicas de TCC, EM e THS.

Embora as atividades grupais sejam incentivadas como práticas fundamentais na promoção de saúde, a formação acadêmica tradicional se apresenta distanciada do paradigma da Saúde Coletiva, ainda compreendendo o processo saúde-doença como predominantemente orgânico, hierarquizado e com estratégias de cuidado centradas na sintomatologia, o que não dá bases suficientes para o trabalho em grupo.

O modelo de atenção presente nos CAPS ad tem como proposta situar a Saúde Mental no campo da Saúde Coletiva, a qual compreende o processo saúde-doença como resultante de processos sociais complexos e que demandam uma abordagem interdisciplinar e intersetorial (Yasui \& Costa-Rosa, 2008). No paradigma psicossocial as práticas devem ser voltadas para a reinserção social e o resgate da cidadania dos usuários, que são mais facilmente enfocados por meio de atividades coletivas (Ferreira Neto, 2008).

Conforme Oliveira, Sartes, Ribeiro, Ismael e Cazassa (2017), a Terapia cognitivocomportamental em grupo (TCCG) vem demonstrando tratar-se de um modelo bastante promissor para o tratamento dos quadros de dependência de substâncias psicoativas. O foco da TCCG para dependência é na psicoeducação, no aprendizado e na implementação de estratégias de enfrentamento para o manejo das situações de risco, prevenção de recaída e manutenção da abstinência.

A respeito do emprego incipiente das estratégias cognitivo-comportamentais em CAPS ad no Brasil, Souza (2010) corrobora que na América Latina, os princípios da TCC apresentam-se ainda restritos às pesquisas nos centros universitários, pois a psicanálise continua a ser a ferramenta mais proeminente nos serviços de saúde, apesar de todas as evidências de eficácia do modelo cognitivo-comportamental (Jhanjee, 2014; McGovern \& Carroll, 2003; Rawson \& Clark, 2017; Roggi et al, 2015).

Diehl, Cordeiro e Laranjeira (2011) apontam vários problemas na organização dos serviços de atenção a usuários de álcool e outras drogas no Brasil e observam que grande parte dos programas de tratamento se organiza de forma empírica a partir do empenho de seus profissionais. A falta da adoção de abordagens baseadas em evidência científica gera serviços com potencial de atendimento terapêutico limitado, com a consequente cronificação de pacientes nos CAPS e alto índice de abandono (Flora, 2008). 
Outro problema verificado é a falta de formação dos profissionais da rede de atenção aos usuários de drogas, visto que as grades curriculares dos cursos de saúde não contemplam adequadamente o tema (Souza, 2010). Além disso, vários estudos evidenciam a falta de investimento em formação continuada dos profissionais do CAPS (Alves, Dourado, \& Côrtes, 2013; Anjos Filho \& Souza, 2017; Cantele, Arpini, \& Roso, 2012).

\section{Considerações}

As estratégias cognitivo-comportamentais apresentam várias vantagens a favor de sua adoção como referência para o tratamento de substâncias psicoativas na política de atenção a usuários de álcool e outras drogas, assim como já ocorre no Programa Nacional de Controle do Tabagismo do Ministério da Saúde no Brasil (Ministério da Saúde, 2001).

Entre as características destas intervenções que se mostram vantajosas à sua inserção na saúde pública, destacamos o fato de serem abordagens estruturadas, diretivas e focais, o que viabiliza sua sistematização em protocolos, facilitando sua aplicação e avaliação de resultados terapêuticos. Além disso, podem ser incorporadas por qualquer profissional de saúde.

Apesar dos benefícios apresentados pelas abordagens cognitivo-comportamentais em pesquisas científicas, persistem obstáculos para transportar estes resultados para a prática nos serviços de Saúde Mental no Brasil. Desse modo, torna-se fundamental uma reflexão crítica e avaliação, com métodos baseados em evidência, das metodologias de tratamento empregadas na atenção psicossocial a usuários de álcool e outras drogas no Brasil.

Embora este estudo tenha contribuído para uma revisão atualizada sobre os tratamentos para álcool e outras drogas e, principalmente, sobre a situação da inserção da prática baseada em evidência pautada na TCC no CAPS ad no Brasil, apresentou algumas limitações. O estudo não se caracterizou por uma revisão sistemática da literatura, especialmente no que se refere à utilização dos descritores específicos de cada base de dados, o que pode ter excluído estudos relevantes para atender o primeiro objetivo.

É necessário avançar no desenvolvimento de instrumentos de políticas públicas que permitam a difusão de boas práticas de intervenções comprovadamente custo-efetivas e acessíveis à população. Sugere-se mais estudos relatando o emprego destas estratégias em CAPS ad, com o intuito de maior compreensão da abordagem neste contexto, a fim de verificar resultados e limitações. 


\section{Referências}

Alves, H. M. C., Dourado, L. B. R., \& Côrtes, V. N. Q. (2013). A influência dos vínculos organizacionais na consolidação dos Centros de Atenção Psicossociais. Ciência $e$ Saúde Coletiva, 18(10), 2965-2975. doi: 10.1590/S1413-81232013001000021

Anjos Filho, N. C., \& Souza, A. M. P. (2017). A percepção sobre o Trabalho em Equipe multiprofissional dos trabalhadores de um Centro de Atenção Psicossocial em Salvador, Bahia, Brasil. Interface (Botucatu), 21(60), 63-76. doi: 10.1590/180757622015.0428

Beck, A. T., Wright, F. D., Newman, C. F., \& Liese, B. S. (1993). Cognitive Therapy of Substance Abuse: Theoretical Rationale. In L. S. Onken, J. D. Blaine, \& J. J. Boren (Eds.), Behavioral Treatments for Drug Abuse and Dependence (pp. 123-146). Rockville: NIDA Research Monograph.

Bello, L. A. (2015). Desafios da transferência da evidência científica para o desenho e implementação de políticas sobre o uso de substâncias psicoativas. In T. M. Ronzani, P. H. A. Costa, D. C. B. Mota, \& T. J. Laport (Orgs.), Redes de Atenção aos Usuários de Drogas: Políticas e práticas (pp. 17-39). São Paulo: Cortez.

Bourguignon, L. N., Guimarães, E. S. \& Siqueira, M. M. (2010). A atuação do enfermeiro nos grupos terapêuticos dos CAPS AD do Espírito Santo. Cogitare enfermagem, 15(3), 467-473. doi: 10.5380/ce.v15i3.18889

Bowen, S., Witkiewitz, K., Clifasefi, S. L., Grow, J., Chawla, N., Hsu, S. H., . . Larimer, M. E. (2014). Relative efficacy of mindfulness-based relapse prevention, standard relapse prevention, and treatment as usual for substance use disorders: A randomized clinical trial. JAMA Psychiatry, 71-5, 547-556. doi: 10.1001/jamapsychiatry.2013.4546

Brasil. Ministério da Saúde. (2001). Abordagem e Tratamento do Fumante. Rio de Janeiro: INCA, Consenso.

Brasil. Ministério da Saúde. (2004). A Política do Ministério da Saúde para Atenção Integral a Usuários de Álcool e outras Drogas. Brasília: Ministério da Saúde.

Brasil (2011). Portaria $n^{o}$ 3088, de 23 de dezembro de 2011. Institui a Rede de Atenção Psicossocial para pessoas com sofrimento ou transtorno mental e com necessidades decorrentes do uso de crack, álcool e outras drogas, no âmbito do Sistema Único de Saúde (SUS). Recuperado de http://bvsms.saude.gov.br/bvs/saudelegis/gm/2011/prt3088_23_12_2011_rep.html 
Cantele, J., Arpini, D. M., \& Roso, A. (2012). A Psicologia no Modelo Atual de Atenção à Saúde Mental. Psicologia Ciência e Profissão, 32(4), 910-925. doi: 10.1590/S141498932012000400011

Chiesa, A., \& Serretti, A. (2014). Are mindfulness-based interventions effective for substance use disorders? A systematic review of the evidence. Subst Use Misuse, 49(5), 492-512. doi: 10.3109/10826084.2013.770027

Diehl, A., Cordeiro, D. C., \& Laranjeira, R. (2011). Organização dos Serviços de Tratamento em Dependência Química. In A. Diehl, D. C. Cordeiro, \& R. Laranjeira (Orgs.), Dependência Química: Prevenção, tratamento e políticas públicas (pp. 507-517). Porto Alegre: Artmed.

Dobson, K. S., \& Scherrer, M. C. (2011). História e futuro das terapias cognitivocomportamentais. In P. Knapp (Ed.), Terapia cognitivo-comportamental na prática psiquiátrica (pp. 42-57). Porto Alegre: Artmed.

Ferreira Neto, J. L. (2008). Práticas Transversalizadas da Clínica em Saúde Mental. Psicologia Reflexão e Crítica, 21(1), 110-118. doi: 10.1590/S010279722008000100014

Flora, F. A. M. (2008). A Crise da Saúde Mental. Revista de Psiquiatria Clínica, 35(1), 3334. Recuperado de https://www.scielo.br/pdf/rpc/v35n1/v35n1a07

Freire, S. D. (2011). Implicações práticas no tratamento psicoterápico da dependência química. In I. Andretta, \& M. S. Oliveira (Orgs.), Manual Prático de Terapia cognitivo-comportamental (pp. 389-402). São Paulo: Casa do Psicólogo.

Jhanjee, S. (2014). Evidence based psychosocial interventions in substance use. Indian journal of psychological medicine, 36(2), 112. doi: 10.4103/0253-7176.130960

Kantorski, L. P., Lisboa, L. M., \& Souza, J. (2005). Grupo de prevenção de recaídas de álcool e outras drogas. SMAD. Revista eletrônica saúde mental álcool e drogas, 1(1), 1-15. Recuperado de http://pepsic.bvsalud.org/scielo.php?script=sci_arttext\&pid=S1806 69762005000100005

Knapp, P. (2004). Princípios fundamentais da terapia cognitiva. In P. Knapp (Coord.), Terapia Cognitivo-Comportamental na Prática Psiquiátrica (pp. 19-41). Porto Alegre: Artmed.

Knapp, P., \& Bertolote, J. M. (1994). Prevenção de recaída: Um manual para pessoas com problemas pelo uso de álcool e de drogas. Porto Alegre: Artes Médicas. 
Larimer, M. E., Palmer, R. S., \& Marlatt, G. A. (1999). Relapse prevention: An overview of Marlatt's cognitive-behavioral model. Alcohol Research and Health, 23(2), 151-160. Recuperado de https://www.ncbi.nlm.nih.gov/pmc/articles/PMC6760427/

Lucena-Santos, P., Pinto-Gouveia, J., \& Oliveira, M. S. (2015). Primeira, Segunda e Terceira Geração de Terapias Comportamentais. In P. Lucena-Santos, J. Pinto-Gouveia, J., \& M. S. Oliveira (Eds), Terapias Comportamentais de Terceira Geração: Guia para profissionais (pp. 29-58). Novo Hamburgo: Sinopsys.

Martins, T. D. (2011). Terapia cognitivo-comportamental em grupo no tratamento do abuso de Benzodiazepínicos (Trabalho de conclusão de curso de Especialização). Universidade Federal do Rio Grande do Sul, Porto Alegre, Brasil. Recuperado de https://www.lume.ufrgs.br/handle/10183/39081

McGovern, M. P., \& Carroll, K. M. (2003). Evidence-based practices for substance use disorders. The Psychiatric Clinics of North America, 26(4), 991. Recuperado de https://www.ncbi.nlm.nih.gov/pmc/articles/PMC3678283/

Miguel, A. Q. C., \& Gaya, C. M. (2013). Técnicas e terapias comportamentais aplicadas ao tratamento da dependência química. In N. A. Zanelatto, \& R. Laranjeira (Org.), $O$ tratamento da dependência química e as terapias cognitivo-comportamentais: Um guia para terapeutas (pp. 313-329). Porto Alegre: Artes Médicas.

O'Farrell, T. J., \& Fals-Stewart, W. (2006). Behavioral couples therapy for alcoholism and drug abuse. New York: The Guilford Press. Recuperado de https://www.ncbi.nlm.nih.gov/pmc/articles/PMC3215582/

Oliveira, M. S., Sartes, L. M. A., Ribeiro, N. S., Ismael, S. M. C., \& Casazza, M. J. (2017). Terapia Cognitivo-comportamental em Grupo Aplicada a Usuários de Drogas. In C. B. Neufeld, \& B. P. Rangé (Orgs.), Terapia Cognitivo-comportamental em Grupos: Das evidências às práticas (pp. 212-235). Porto Alegre: Artmed.

Project MATCH Research Group. (1997). Matching Alcoholism Treatments to Client Heterogeneity: Project MATCH posttreatment drinking outcomes. J Stud Alcohol, 58(1), 7-29. Recuperado de https://pubmed.ncbi.nlm.nih.gov/8979210/

Rangé, B. P., Falcone, E. M. O., \& Sardinha, A. (2007). História e panorama atual das terapias cognitivas no Brasil. Revista Brasileira de Terapias Cognitivas, 3(3), 1-22. Recuperado de http://pepsic.bvsalud.org/scielo.php?script=sci_arttext\&pid=S180856872007000200006

Rawson, R. A., \& Clark, N. (2017). Interventions for the management of substance use disorders: An overview. Eastern Mediterranean Health Journal, 23(3), 214-221. 
Recuperado de http://www.emro.who.int/emhj-volume-23-2017/volume-23-issue3/interventions-for-the-management-of-substance-use-disorders-an-overview.html

Roggi, P. M. S., Gama, M. F. N., Neves, F. S., \& Garcia, F. (2015). Update on treatment of craving in patients with addiction using cognitive behavioral Therapy. Clinical Neuropsychiatry, 12(5), 118-127. Recuperado de https://www.clinicalneuropsychiatry.org/download/update-on-treatment-of-craving-inpatients-with-addiction-using-cognitive-behavioral-therapy/

Rollnick, S., Miller, W. R., \& Butler, C. C. (2009). Entrevista Motivacional no Cuidado à Saúde: Ajudando os pacientes a mudar o comportamento. Porto Alegre: Artmed.

Romanini, M., Pereira, A. S., \& Dias, A. C. G. (2016). Grupo de prevenção de recaídas como dispositivo para o tratamento da dependência química. Disciplinarum Scientia: Ciências da Saúde, 11(1), 115-132. Recuperado de https://periodicos.ufn.edu.br/index.php/disciplinarumS/article/view/970/913

Roozen, H. G., Boulogne, J. J., Tulder, M. W., Brink, W., Jong, C. A. J. \& Kerkhof, A. J. F. M. (2004). A systematic review of the effectiveness of the community reinforcement approach in alcohol, cocaine and opioid addiction. Drug and Alcohol Dependence, 74(1). 1-13. doi: 10.1016/j.drugalcdep.2003.12.006

Santos, E. G. D. (2010). O grupo como estratégia terapêutica nos Centros de Atenção Psicossocial Álcool e Drogas do Espírito Santo (Dissertação de mestrado). Centro de Ciências da Saúde da Universidade Federal do Espírito Santo, Vitória, Espírito Santo, Brasil. Recuperado de http://portais4.ufes.br/posgrad/teses/tese_3941_.pdf

Silva, C. J., \& Serra, A. M. (2004). Terapias Cognitiva e Cognitivo-Comportamental em dependência química Cognitive and Cognitive-Behavioral Therapy for substance abuse disorders. Revista Brasileira de Psiquiatria, 26(Supl. 1), 33-39. doi: 10.1590/S1516-44462004000500009

Silva, S. S., Pereira, R. C., \& Aquino, T. A. A. A. (2011). Terapia Cognitivo-Comportamental no Ambulatório Público: Possibilidades e desafios. Revista Brasileira de Terapias $\begin{array}{llll}\text { Cognitivas, } & 7(1), & 44-49 . & \text { Recuperado }\end{array}$ http://pepsic.bvsalud.org/scielo.php?script=sci_arttext\&pid=S180856872011000100008

Souza, I. C. W. (2010). O Profissional da APS e as Substâncias de Abuso: O papel da capacitação na rotina da assistência (Dissertação de Mestrado). Faculdade de Medicina da Universidade Federal de Juiz de Fora, Minas Gerais, Brasil. Recuperado 
de https://www.ufjf.br/crepeia/files/2009/09/profissional-de-saude-substancias-abusocapacitacao.pdf

Siqueland, L., \& Crits-Christoph, P. (1999). Current developments in psychosocial treatments of alcohol and substance abuse. Current Psychiatry Reports, 1(2), 179-184. doi: 10.1007/s11920-999-0029-6

Yasui, S., \& Costa-Rosa, A. (2008). A estratégia atenção psicossocial: Desafio na prática dos novos dispositivos de Saúde Mental. Saúde em Debate, 32(78, 79, 80), 27-37. Recuperado de https://www.redalyc.org/articulo.oa?id=406341773003

\section{Endereço para correspondência}

\section{Érica Aparecida Schefer de Almeida}

ICH - Departamento de Psicologia

Rua José Lourenço Kelmer, s/n, Cidade Universitária, Juiz de Fora - MG, Brasil. CEP 36036-900

Endereço eletrônico: ericaasalmeida@ yahoo.com.br

\section{Laisa Marcorela Andreoli Sartes}

ICH - Departamento de Psicologia

Rua José Lourenço Kelmer, s/n, Cidade Universitária, Juiz de Fora - MG, Brasil. CEP 36036-900

Endereço eletrônico: laisa.sartes@gmail.com

Recebido em: 25/05/2019

Reformulado em: 24/10/2020

Aceito em: 18/11/2020

\section{Notas}

* Graduação em Psicologia pelo CES - JF (2006). Formação em TCC pelo NEISME (2008). Mestrado em Psicologia pela UFJF (2019). Psicóloga do CAPS I de Matias Barbosa (2011-atual).

** Psicóloga (UFJF), mestre e doutora em Ciências pela UNIFESP. Professora associada do Departamento de Psicologia da UFJF desde 2011.

Este artigo de revista Estudos e Pesquisas em Psicologia é licenciado sob uma Licença Creative Commons Atribuição-Não Comercial 3.0 Não Adaptada. 